\title{
Synthesis of Modified Oligonucleotides and Conjugates
}

\section{INTRODUCTION}

$\mathbf{T}$ he genetic information encoded in either genomic DNA or transcribed mRNAs has become an enticing target for chemical modifications and potential therapeutic indications. Specifically, the search for methods that selectively prevent expression of unwanted genes has been actively pursued for more than a decade. Theoretically, it is possible to exploit the selective Watson-Crick hydrogen bond formation between complementary nucleic acid strands to control gene expression either by preventing transcription of genomic DNA (an antigene strategy) or by interfering with the translation of mRNAs (an antisense strategy) to gene products. With the advent of rapid and efficient methods for automated synthesis of oligonucleotides, it has indeed been possible to prepare modified oligonucleotides complementary to specific nucleotide sequences of mRNA targets. As a consequence of binding, and on the basis of particular cellular mechanisms, these oligonucleotides can disrupt the flow of genetic information from transcribed mRNAs to proteins. Thus, gene expression can be controlled at the mRNA level by modified oligonucleotides, and this antisense strategy has led, over the years, to the development of therapeutic oligonucleotides against cancer and various infectious diseases in humans. In this context, UNIT 4.1 reports recent advances in the application of oligonucleotides as drug candidates, describes the relationship between oligonucleotide modifications and their therapeutic profiles, and provides general guidelines for enhancing oligonucleotide drug properties.

Along similar lines, modern methods for the chemical synthesis of oligonucleotides have facilitated the covalent attachment of reporter and conjugate groups to DNA and RNA. Such modifications generate properties not normally present in native DNA or RNA oligonucleotides. For example, addition of a conjugate group to an oligonucleotide can be used to alter its solubility, charge, binding affinity, spectroscopic properties, and function. Furthermore, oligonucleotide conjugates can provide structural information on the nature of specific nucleic acid complexes such as protein-DNA(RNA) or ligandDNA(RNA) complexes. Unlike enzymatic methods, chemical methods for the incorporation of modified nucleosides into oligonucleotides permit a precise control over the type, number, and placement of the reporter and conjugate groups within the sequence of interest. Typically, fluorescent markers, intercalators, cross-linkers, and DNA/RNA cleaving agents are added either to the $5^{\prime}$ or $3^{\prime}$ terminus of oligonucleotides, or inserted anywhere within an oligonucleotide sequence. These oligonucleotide conjugates can then be specifically dedicated to diagnostic or therapeutic applications. UNIT 4.2 presents an overview of the methods employed for the attachment of reporter and conjugate groups to the $5^{\prime}$ terminus of oligonucleotides. The unit lists several reporter and conjugate groups that can be directly incorporated into oligonucleotides mainly via ligand phosphoramidite or $H$-phosphonate derivatives. This knowledge is reduced to practice in UNIT 4.3, which delineates step-by-step protocols for the synthesis of intercalating and photo-cross-linking ligand phosphoramidites as well as their addition to the $5^{\prime}$ terminus of oligonucleotides.

Contributed by Serge L. Beaucage

Current Protocols in Nucleic Acid Chemistry (2004) 4.0.1-4.0.3

Copyright $(\subset 2004$ by John Wiley \& Sons, Inc.

Synthesis of Modified Oligonucleotides and Conjugates

4.0.1

Supplement 17 
Reporter and conjugate groups are, however, not always stable to the conditions used during either oligonucleotide synthesis or the subsequent deprotection steps. Consequently, the incorporation of these groups into oligonucleotides must be accomplished according to an "indirect" approach. This method involves functionalization of the $5^{\prime}$ terminus of oligonucleotides, mostly with nucleophilic groups. Although the indirect approach to the preparation of oligonucleotide conjugates is extensively reviewed in UNIT 4.2, procedures for the modification of oligonucleotides at the $5^{\prime}$ terminus are outlined in UNIT 4.9. Following deprotection and purification, the functionalized oligonucleotides are then conjugated to selected electrophilic reporter or conjugate groups. Methods describing the incorporation of functional groups into ligands are reported in UNIT 4.8, and methods for conjugation of these ligands to the $5^{\prime}$-functionalized oligonucleotides are described in UNIT 4.10.

An attractive method for the preparation of oligonucleotide conjugates consists of functionalizing the $5^{\prime}$-terminus of solid-phase-bound oligonucleotides with a diene phosphoramidite derivative followed by conjugation of the deprotected oligonucleotides with selected dienophile reporter groups via a Diels-Alder cycloaddition reaction. The details of this convenient conjugation method are provided in UNIT 4.18. For similar applications, a facile and efficient $5^{\prime}$-iodination of solid-phase-linked oligonucleotides is presented in UNIT 4.19. 5'-Iodo oligonucleotides allow conjugation with small molecules or macromolecules having a strongly nucleophilic group. While the functionalization of oligonucleotides at either the $5^{\prime}$ - or $3^{\prime}$-terminus is invaluable in the preparation of oligonucleotide conjugates, the incorporation of uridine $2^{\prime}$-carbamates into oligonucleotides nicely complements these methods for direct or indirect attachment of various ligands to DNA or RNA. This versatile method is carefully detailed in UNIT 4.21.

The purification of synthetic oligonucleotides from a mixture of shorter sequences is always a concern. Thus, purification methods that can easily provide oligonucleotides of high purity are of great value. UNIT 4.20 highlights a reversible biotinylation of the $5^{\prime}$-terminus of oligonucleotides, which allows simple and highly effective affinity purification of these biomolecules.

This chapter is also intended to include overviews and step-by-step protocols addressing the attachment of reporter and conjugate groups to the nucleobase, the $3^{\prime}$ terminus, and the carbohydrate portion of oligonucleotides for diagnostic applications. In this regard, UNIT 4.5 surveys a number of conjugation methods for oligonucleotides displaying various $3^{\prime}$ functional groups, and UNIT 4.6 describes the step-by-step preparation of $3^{\prime}$-aminoalkylated or $3^{\prime}$-mercaptoalkylated oligonucleotides, and their respective conjugation to fluorescent reporter groups. Other protocols describing the preparation of oligonucleotides with modified nucleobases, sugars, and internucleotide linkages for potential therapeutic applications will also be incorporated into this chapter to provide a comprehensive coverage of these cutting edge technologies. In this context, UNIT 4.4 delineates the solid-phase synthesis of chimeric 2-5A-DNA oligonucleotide conjugates along with the preparation of the phosphoramidite monomers used for such synthesis. In addition, the synthesis and purification of oligonucleotides composed of $\mathrm{N}^{\prime} \rightarrow \mathrm{P}^{\prime}$ phosphoramidate and either phosphodiester or phosphorothioate linkages, as presented in UNIT 4.7, is a relevant example of such protocols. UNIT 4.11 provides manual and automated methods for the synthesis of peptide nucleic acids (PNAs), whereas UNIT 4.12 details the synthesis of locked nucleic acids (LNAs). These modified nucleic acids exhibit high affinity for complementary RNA and DNA strands, and are thus amenable to a wide range of biomedical applications. In this regard, a procedure for the incorporation of LNAs into cells is reported in UNIT 4.13. The conjugation of peptides to oligonucleotides to generate nucleopeptides is the focus of intense scrutiny given the reported abilities of these conjugates at enhancing intracellular delivery of therapeutic oligonucleotides. However, the preparation 
of peptide-oligonucleotide conjugates is challenging. An inspiring method for the stepwise solid-phase synthesis of nucleopeptides is delineated in UNIT 4.22, thus making these bioconjugates more available for further cell delivery studies.

Synthetic nucleic acids containing vicinal $2^{\prime}, 5^{\prime}$ - and $3^{\prime}, 5^{\prime}$-phosphodiester linkages have been useful to investigations related to pre-mRNA splicing events and to studies assessing the specificity of RNA lariat debranching enzymes. These branched nucleic acids may also find applications in the development of biosensors and diagnostics, or may serve as "molecular anchors" in the formation of triplex and tetraplex structures. Two synthetic strategies for the preparation of branched oligonucleotides are outlined and developed in UNIT 4.14.

Transfer RNAs (tRNAs) incorporate a variety of modified nucleobases, which are known to affect the biological activity of tRNAs through structural changes. To better understand the role of these modified nucleobases, UNIT 4.23 reports on the synthesis of ribonucleoside phosphoramidites functionalized with either 6-methylthio purine or 2-methylthio-6chloro purine for incorporation into relatively short RNA oligonucleotide models. Following oligonucleotide synthesis using these phosphoramidites, the modified nucleobases are chemically converted to a number of native tRNA nucleobase modifications to further studies in this research area.

Modified nucleic acids such as $2^{\prime}$-deoxy-2'-fluoro- $\beta$-D-oligoarabinonucleotides are unique in that they form more stable duplex structures with RNA complements than identical unmodified DNA:RNA duplexes. In addition, the modified heteroduplexes are substrates for RNase $\mathrm{H}$, an enzyme viewed by many as the major effector of antisense activity. Thus, given the promising antisense properties of $2^{\prime}$-fluoroarabinonucleic acids, methods for the synthesis of $2^{\prime}$-deoxy- $2^{\prime}$-fluoro- $\beta$-D-arabinonucleoside phosphoramidites and their use in the automated synthesis of $2^{\prime}$-fluoroarabinonucleotides are included in UNIT 4.15.

Phosphorothioated oligonucleotides have also found therapeutic applications in antisense settings. Such modified oligonucleotides are chiral at phosphorous, and those exhibiting the $S_{\mathrm{p}}$ configuration are significantly more resistant to nucleases found in human plasma than $P$-diastereomeric or $R_{\mathrm{p}}$-phosphorothioated oligonucleotides. Methods for the synthesis of oligonucleotides with stereodefined phosphorothioate linkages would therefore be valuable in the preparation of therapeutic oligonucleotides with optimal resistance to nucleases. UNIT 4.17 specifically describes the use of deoxyribonucleoside-3'-O-(2-thio1,3,2-oxathiaphospholane) derivatives in the $P$-stereocontrolled synthesis of phosphorothioated oligonucleotides.

Another class of modified oligonucleotides with potential biomedical applications relates to phosphorothiated oligodeoxyribonucleotides expressing specific $\mathrm{CpG}$ motifs. These oligonucleotides strongly stimulate B cells, natural killers cells, and antigen-presenting cells to proliferate and/or secrete cytokines, chemokines, and immunoglobulins. Given that a number of structural factors influences the recognition of $\mathrm{CpG}$-oligonucleotides and expression of their immunostimulatory properties, UNIT 4.16 provides an overview of the chemical strategies used to assess the significance of these factors toward the development of human therapies.

Serge L. Beaucage

Synthesis of Modified Oligonucleotides and Conjugates

4.0.3

Supplement 17 